\title{
INFARTO HEMORRÁGICO TESTICULAR EN NEONATO: PRESENTACIÓN DE UN CASO
}

\author{
B. CORONEL SÁNCHEZ, C. DOMÍNGUEZ HINAREJOS, A. SERRANO DURBÁ, \\ M. MARTÍNEZ VERDUCH, F. ESTORNELL MORAGUES, F. GARCÍA IBARRA
}

Servicio de Urología Infantil. Hospital Infantil La Fe. Valencia.

Actas Urol Esp. 28 (5): 393-395, 2004

\section{RESUMEN}

INFARTO HEMORRÁGICO TESTICULAR EN NEONATO: PRESENTACIÓN DE UN CASO

El infarto testicular hemorrágico es una entidad poco usual en el neonato, desarrollándose generalmente como consecuencia de la torsión del cordón espermático. Presentamos un caso de infarto testicular en neonato asociado a hidrocele a tensión.

PALABRAS CLAVE: Testículo. Infarto. Hidrocele.

\section{ABSTRACT \\ HEMORRHAGIC INFARCTION OF THE TESTICLE IN THE NEWBORN INFANT. \\ A CASE PRESENTATION}

Hemorrhagic infarction of the testicle is an unusual occurrence in the newborn infant. It usually develops as a consequence of torsion of the spermatic cord. We report a case of global testicular infarction in a newborn associated with a tense hydrocele.

KEY WORDS: Testis. Infarction. Hydrocele.

$\mathrm{E}^{\prime}$ 1 infarto hemorrágico testicular en el periodo neonatal es poco frecuente ${ }^{1,2}$. En la mayoría de los casos, se produce como consecuencia de la torsión extravaginal del cordón espermático ${ }^{1-3}$. Sin embargo, esta patología ha sido descrita en ausencia de torsión, relacionada con traumatismos del parto ${ }^{2-4}$, como complicación de una hernia inguino-escrotal estrangulada ${ }^{1}$ o como infarto idiopático ${ }^{1-3}$.

Presentamos un nuevo caso de infarto hemorrágico testicular unilateral en un neonato, considerando como probable factor desencadenante la presencia de hidrocele a tensión que, por compresión, provocó daño vascular irreversible.

\section{CASO CLÍNICO}

Varón que nace en la 40 semana de gestación con parto vía vaginal y presentación cefálica. El peso al nacer es de $3.700 \mathrm{gr}$ y la talla $47 \mathrm{~cm}$. Se solicita valoración urológica ante la existencia de gran aumento de tamaño escrotal bilateral.

En la exploración física, se objetiva hidrocele a tensión en hemiescroto derecho e hidrocele leve el lado izquierdo, con transiluminación positiva (Fig. 1). El teste izquierdo es de características normales en cuanto a morfología y consistencia, siendo dificultosa la palpación testicular derecha por la presencia de hidrocele a tensión. 


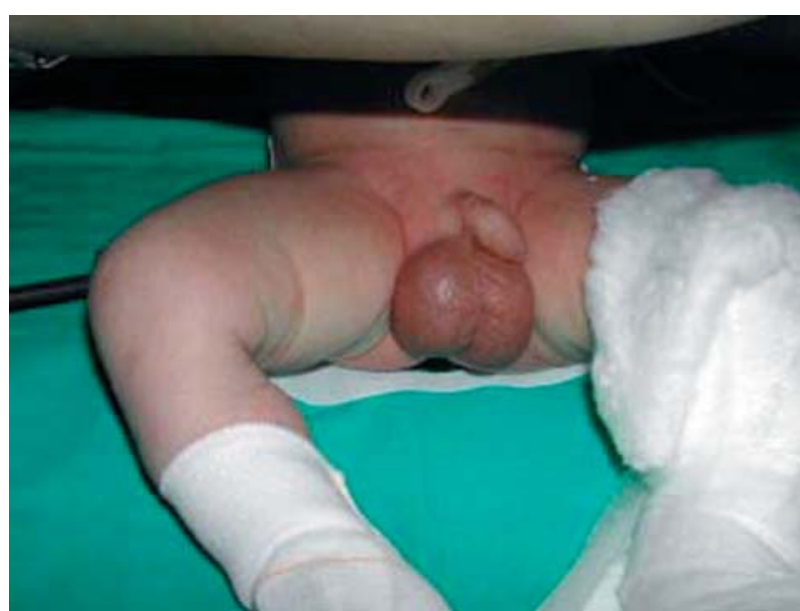

FIGURA 1. Hidrocele bilateral, a tensión en hemiescroto derecho.

En la analítica sanguínea destaca moderada leucocitosis. El sedimento de orina fue normal. La ecografía doppler se realizó ajustando a los niveles más bajos del ecógrafo tanto la frecuencia de pulso como el filtro de pared. Los hallazgos fueron de hidrocele bilateral, siendo de gran tamaño el del lado derecho, presentando el teste derecho flujo doppler arterial conservado, aunque con un índice de resistencia (IR) elevado (IR=0,82; Normal: 0,48-0,75), ausencia de flujo venoso y ecogenicidad heterogénea, sugiriendo áreas de desestructuración. El testículo izquierdo es normal ecográficamente, presentando una velocidad de pico sistólica de $11 \mathrm{~cm} / \mathrm{s}$ y diastólica de $5 \mathrm{~cm} / \mathrm{s}$, siendo el IR de 0,55.

Ante la sospecha de compromiso vascular testicular se decide exploración quirúrgica a las dieciocho horas del nacimiento. Apreciamos hidrocele a tensión sin objetivar torsión del cordón (Fig. 2), apareciendo el teste de coloración violácea y aspecto necrótico, por lo que se realizó orquiectomía (Fig. 3).

La evolución post-operatoria se desarrolló sin complicaciones inmediatas.

La anatomía patológica de la pieza fue de infarto hemorrágico testicular (Fig. 4).

A los ocho meses de seguimiento el paciente se encuentra asintomático habiendo desaparecido el hidrocele izquierdo.

\section{DISCUSIÓN}

La incidencia del infarto hemorrágico testicular es baja en cualquier época de la vida. En adultos,

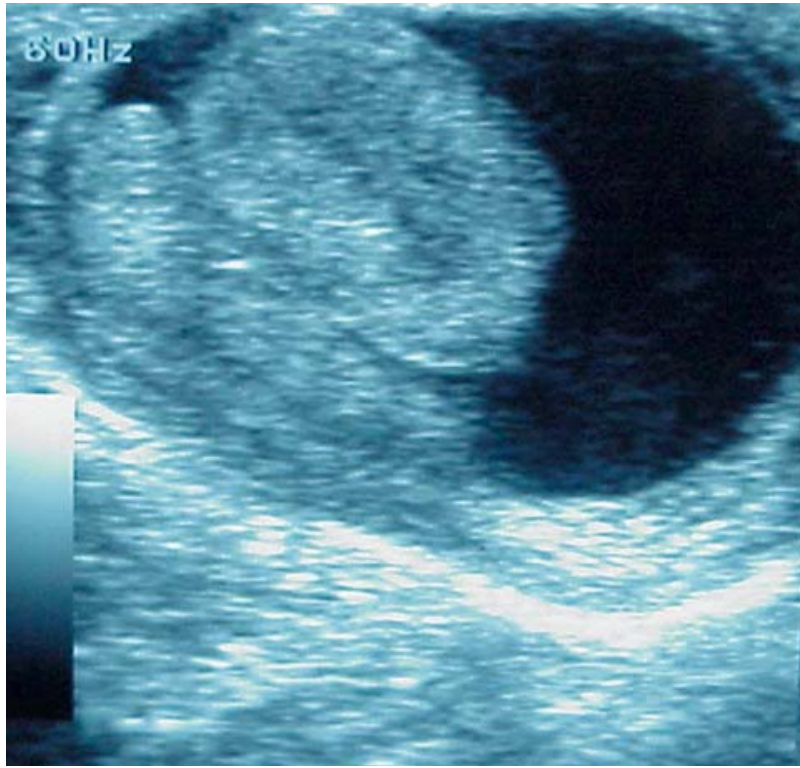

FIGURA 2. Hidrocele y ausencia de torsión del cordón espermático.

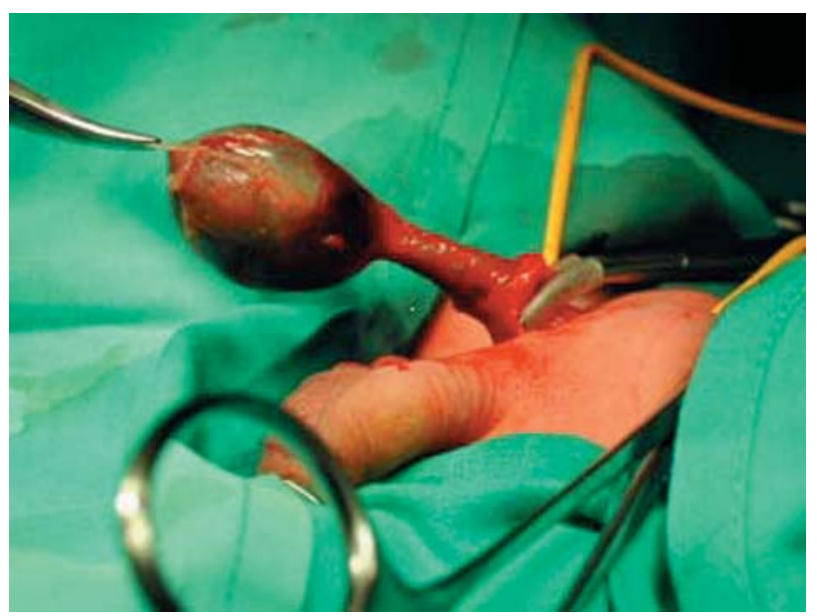

FIGURA 3. Testículo necrótico.

suele estar relacionado con la torsión intravaginal del cordón espermático, epidídimo-orquitis severas, post-herniorrafia o traumatismos escrotales $^{5-8}$.

En neonatos se ha descrito en relación a la torsión extravaginal del cordón espermático y como complicación de una hernia estrangulada ${ }^{1,2}$.

La hipótesis del traumatismo obstétrico es planteada por diversos autores en la literatura revisada $^{2-4}$. Se consideran factores predisponentes de lesiones en la región perineal, la presentación obstétrica de nalgas, la utilización de fórceps y el peso elevado al nacimiento ${ }^{4,9}$. 


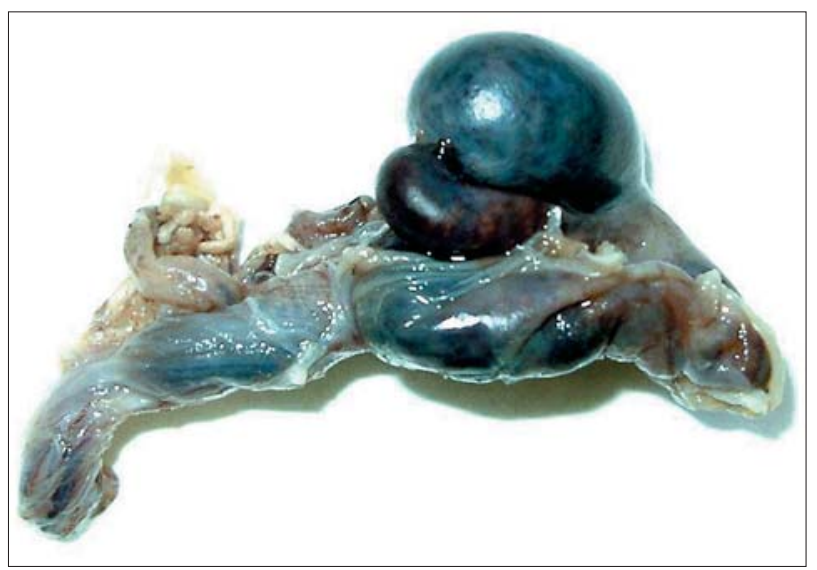

FIGURA 4. Necrosis hemorrágica testicular.

Ney y cols. ${ }^{7}$ presentan un caso en el que el estudio doppler testicular en presencia de gran hidrocele a tensión en un adulto de 48 años objetiva disminución severa del flujo diastólico y desaparición del flujo venoso, lo que sugiere que puede existir compromiso del retorno venoso y predisponer al infarto testicular, como demuestra la evolución, con normalidad de la vascularización testicular tras punción del hidrocele.

Todas estas patologias provocarían procesos de compresión venosa, trombosis del plexo pampiniforme y posterior infarto por congestión ${ }^{3,7,10}$.

No hemos encontrado en la literatura revisada ningún caso de infarto testicular en la edad pediátrica asociado a hidrocele a tensión como el caso que describimos. Pensamos que la compresión provocada por el hidrocele fue el factor desencadenante de la injuria vascular.

Concluimos que el hidrocele debería ser una entidad a tener en cuenta como factor etiológico del infarto testicular. Ante la sospecha de compromiso vascular, debería procederse a la exploración quirúrgica precoz o al vaciamiento del hidrocele como primera medida seguido de cirugía correctora, a fin de evitar la pérdida del testículo.

\section{REFERENCIAS}

1. FERNICOLA AR.: Idiopathic hemorrhagic infarction of the testicle in the newborn. J Urol 1954; 72: 230.

2. RAVICH R.: Hemorrhagic infarction of the testicle in the newborn infant. J Urol 1947; 57: 875.

3. PENSON D, ARONSON W.: Segmental testicular infarction in the neonate: a case report. J Urol 1995; 153: 1992-1993.

4. MATHEWS R, SHERIDAN M.E, PATIL U.: Neonatal testicular loss secondary to perineal trauma in breech presentation. BJU Int 1999; 83: 1069-1070.

5. SRIPRASAD S, KOOIMAN G, MUIR G, SIDHU P.: Acute segmental testicular infarction: differentiation from tumour using high frequency colour doppler ultrasound. B J Radiol 2001; 74: 965-967.

6. SHY-CHYI C, CHING-JIUNN W, ANN C, HAI-SUNG H.: Segmental hemorrhagic infarction of testis associated with epididymitis. J Clin Ultrasound 1998; 26(6): 326-327.

7. NYE P, PRATI R.: Idiopathic hydrocele and absent testicular diastolic flow. J Clin Ultrasound 1997; 25 (1): 43-46.

8. FITZGERALD SW, ERICKSON S, DE WIRE DM, FOLEY WD, LAWSON TL, BEGUN FF, LAWSON RK.: Color Doppler sonography in the evaluation of the adult acute scrotum. J Ultrasound Med 1992; 11 (10): 543-548.

9. TIWARY CM.: Testicular injury in breech delivery: possible implications. Urology 1989; 34: 210-212.

10. COSTA M, CALLEJA R, BALL RY, BURGESS N.: Segmental testicular infarction. BJU Int; 83: 525.

Dra. B. Coronel Sánchez

Servicio de Urología

Hospital Universitario Dr. Peset

Avda. Gaspar Aguilar, 90

46017 Valencia

(Trabajo recibido 1 julio de 2003) 